\title{
Attention to the hands disrupts skilled typewriting: The role of vision in producing the disruption
}

\author{
Kristy M. Tapp • Gordon D. Logan
}

Published online: 14 September 2011

(C) Psychonomic Society, Inc. 2011

\begin{abstract}
Drawing typists' attention to their hands by asking them to type only letters assigned to the left or the right hand disrupts their performance, slowing the rate of typing and increasing errors. In this article we test the hypothesis that slowing occurs because typists watch their hands to determine which hand types which letter. Skilled typists were cued to type letters of one hand or of both hands while they could view their hands on the keyboard and while their vision was blocked by a box placed over the keyboard. Typing was slower when letters of one hand were typed than when letters of both hands were typed, and the slowing was greater when the hands were covered than when they were not. This supports the hypothesis that slowing occurs because typists watch their hands. However, typists were able to type letters of one hand when the keyboard was covered, so typists must have monitored kinesthetic information as well.
\end{abstract}

Keywords Hierarchical control · Automaticity ·

Monitoring · Typewriting

From William James (1890) to Lashley (1951), psychologists have described a paradox in skilled performance: Experts execute skills fluently but have little explicit knowledge of how they produce their actions. Recent studies demonstrated this paradox in typewriting. Skilled typists produce 5-6 keystrokes per second but know little about the locations of the keys on the keyboard (Liu, Crump, \& Logan, 2010) or which hand types each letter (Logan \& Crump, 2009). Psychologists have also described

K. M. Tapp $(\bowtie) \cdot$ G. D. Logan

Department of Psychology, Vanderbilt University,

Nashville, TN 37240, USA

e-mail: kristy.m.tapp@vanderbilt.edu a corollary paradox, that drawing attention to the execution of skills disrupts skilled performance (e.g., Beilock, Carr, MacMahon, \& Starkes, 2002). Making implicit knowledge explicit imposes a cost. This corollary is also apparent in typewriting. Skilled typing is much slower and error prone if typists are asked to type only the letters assigned to one hand (Logan \& Crump, 2009).

A popular resolution to these paradoxes is to propose that skills are controlled hierarchically, with the processing of explicit knowledge occurring at higher levels and the processing of implicit knowledge at lower levels (Lashley, 1951; Logan \& Crump, 2011; Shaffer, 1976). Making implicit knowledge explicit is costly because lower-level processes must change to allow higher-level processes to access the details. Logan and Crump (2009) suggested that typing only letters assigned to one hand is disruptive because higher-level processes do not know which hand types which letter. They must discover it by watching the hands as they type and inhibit inappropriate keystrokes as they occur. Lower-level processes must slow the rate at which keystrokes are struck to allow higher-level processes enough time to see the keystrokes and inhibit them before they are struck (Logan, 1982). If lower-level processes are not slow enough, higher-level processes may fail to inhibit inappropriate keystrokes. Thus, typing speed is slower and the error rate is higher when typists must type only the letters assigned to one hand.

For this article, we assessed the role of vision in monitoring the hands to inhibit inappropriate keystrokes by covering the hands and keyboard while typists typed only the letters assigned to one hand. Logan and Crump (2009) speculated that vision was important, but they did not assess its role directly. Our experiment provided a direct test. Typists typed single words presented on a screen. Each word was preceded by a "whole" cue (Both) that told them 
to type all letters using both hands, as in normal typewriting, or a "hand" cue (Left or Right) that told them to type only the letters assigned to one hand. Logan and Crump found that typing was slower with hand cues than with whole cues. Our innovation here was to test the role of vision by covering the hands and the keyboard with a box to prevent typists from seeing which hand typed which letter. We covered the hands and keyboard on half of the trials and left them uncovered for the other half. If typists use vision to determine which hand types which letter, the difference between handcue and whole-cue trials should be larger when the keyboard is covered than when it is not. If typists rely on vision entirely, covering the keyboard should prevent them from performing the task. If typists can perform adequately with the keyboard covered, they must rely on nonvisual feedback to determine which hand types each letter. Kinesthesis is a likely possibility (Crump \& Logan, 2010b; Salthouse, 1986), although we did not test its role directly.

Our experiment also assessed the role of vision in normal typing with both hands. Previous research had shown that skilled typists rely on both vision and kinesthesis (Long, 1976; Rabbit, 1978), but that research was done long ago with professional typists. Since then, typewriting has become ubiquitous in Western culture, and many people type as well as the professional typists of yesteryear (Logan \& Crump, 2011). Professional typists mostly transcribed texts and were taught not to look at the keyboard. Modern typists mostly compose texts or type from memory (passwords, user IDs, search engine keywords), and looking at the keyboard is not prohibited. Many modern typists use laptop computers, so their hands are close to the screen and available in peripheral vision as they type. Thus, modern typists may rely more on vision than professional typists did in normal typing, so it is important to replicate the classic studies with modern typists. Long (1976) and Rabbitt (1978) covered the keyboard, as did we.

\section{Method}

Subjects

We recruited 24 typists who had formal training and reported their ability to type 40 words per minute (WPM) or better. They averaged 12.1 years of experience typing and averaged 78 WPM on a typing test (Logan \& Zbrodoff, 1998; range: 41.5-119.1 WPM) with hands uncovered (mean accuracy $=93.4 \%$, range: $83.9 \%-100 \%$ ). They received course credit or $\$ 12$ for $1 \mathrm{~h}$ of participation.

Apparatus and stimuli

A pool of 144 four-letter words was compiled from the MRC Psycholinguistic Database. There were 72 unimanual words (typed with one hand in standard touch typing): 36 were typed with the left hand and 36 with the right hand. There were 72 bimanual words (typed with both hands), 12 in each of six categories: RRLL, RLLR, RLRL, LLRR, LRRL, and LRLR. The unimanual $(M=73.6, S D=180.4)$ and bimanual $(M=191.7, S D=661.2)$ words did not differ in word frequency (Davies, 2008), $t(142)=0.4$.

The experiment was conducted on a personal computer programmed in METACARD using a 15-in. SVGA monitor. Viewing distance was about $57 \mathrm{~cm}$. Responses were recorded on a standard QWERTY keyboard. Cues and target words were displayed on a $24.1 \times 19.7 \mathrm{~cm}$ gray window on a black screen. The cue (Left, Right, or Both) appeared $3.8 \mathrm{~cm}$ below the top of the window, the target appeared $2.5 \mathrm{~cm}$ below the cue, and subjects' responses were echoed $5.1 \mathrm{~cm}$ below the target. Words and cues were presented centrally in black 40-point Helvetica font. For half of the experiment, subjects' hands and the keyboard were covered by a $10.8 \times 27.9 \times 44.5 \mathrm{~cm}$ box that did not constrain hand movement.

\section{Procedure}

Each trial began with a whole or a hand cue. A fixation point appeared 1,000 ms later in the position that the target would occupy. After $500 \mathrm{~ms}$, the fixation point was replaced by the target word. The cue and target word remained on the screen until the spacebar was pressed. Subjects were instructed to type only the letters assigned to the cued hand(s) as quickly and accurately as possible and then to press the space bar.

There were two blocks of 288 trials, with breaks every 72 trials. The keyboard was covered for one block and uncovered for the other, with the order counterbalanced between subjects. Each word was presented four times in random order, once with Right, once with Left, and twice with Both. After each block, subjects completed a typing test (Logan \& Zbrodoff, 1998) to verify their typing speed. Their hands were covered following covered blocks and uncovered following uncovered blocks.

\section{Results}

Figure 1 plots mean response times (RTs) collapsed across subjects for each keystroke relative to the onset of the target word as a function of its position in the word, for each combination of cue type (hand vs. whole) and word type (unimanual vs. bimanual) with the keyboard covered or uncovered. Our analysis focused primarily on typing rate, defined as the slope of the linear function relating RT to position in the word. For whole-cue and for unimanual hand-cue words, the rate corresponded to the interkeystroke 


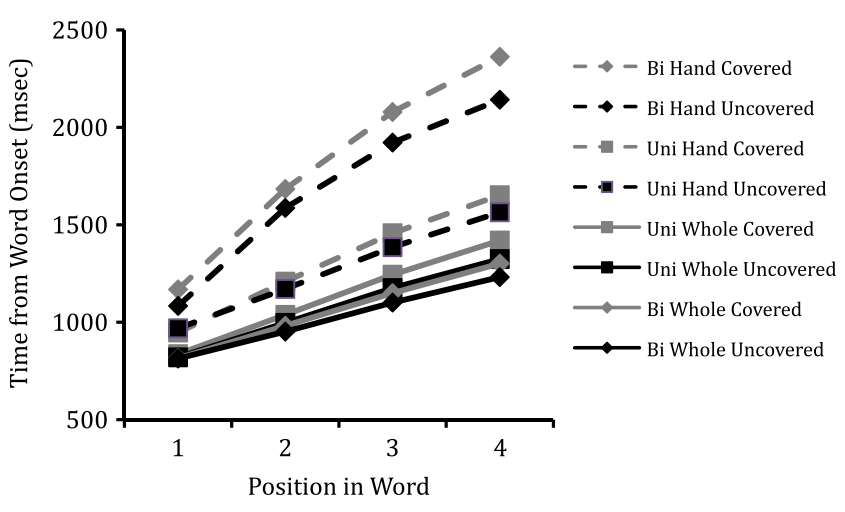

Fig. 1 Mean response times with the hands covered (gray) and uncovered (black) for each keystroke, relative to the onset of the target word, for each cue type (whole, hand) and word type (Uni = unimanual, $\mathrm{Bi}=$ bimanual)

interval (IKSI). For bimanual hand cue words, we plotted mean RTs for each keystroke as a function of the position it occupied in the word and calculated the slope of the function relating RT to position in the word. Thus, with Right as the cue, an RLLR word would contribute to Positions 1 and 4, and an LRRL word would contribute to Positions 2 and 3. We obtained RTs for each position in the word by collapsing over bimanual word types. We conducted a 2 (keyboard: covered vs. uncovered) $\times 2$ (cue type: hand vs. whole) $\times 2$ (word type: unimanual vs. bimanual) ANOVA on typing rates, RTs for the first keystroke (RT1), space bar RTs (SBRT), and error rates. The summary tables for the ANOVAs are presented in Table 1.

Typing rates were $129 \mathrm{~ms} /$ keystroke slower on hand-cue trials than on whole-cue trials. The difference was greater for bimanual words than for unimanual words (223 vs. $36 \mathrm{~ms} /$ keystroke). These findings replicate Logan and Crump (2009), supporting the hypothesis that lower-level processes slow down to allow higher-level processes to monitor which hand types each letter.
Covering the keyboard slowed the typing rate by $33 \mathrm{~ms} /$ keystroke. This increase was greater for hand cues than for whole cues (41 vs. $24 \mathrm{~ms} /$ keystroke). These effects support the hypothesis that typists watch their hands to discover which hand types which letter. However, typists were able to perform the task adequately when the hands were covered, so vision is not absolutely necessary. They may also use kinesthesis (Salthouse, 1986).

Covering the keyboard slowed the typing rate by $24 \mathrm{~ms} /$ keystroke on whole-cue trials, $t(23)=4.6, p<.001$, and slowed speed on the typing test by $3.3 \mathrm{WPM}, t(23)=2.91$, $p<.01$. These effects suggest that modern typists normally depend on visual feedback, like the typists of yesteryear (Salthouse, 1986).

To assess differences in skill, we correlated WPM on the speed test with the effects in the ANOVA on typing rates, calculated as contrasts using 1 and -1 as contrast weights. All correlations were negative, indicating smaller effects with greater skill, but only three correlations were significant: one involving the difference between bimanual and unimanual words, $r(22)=-.46, p<.05$; one involving the interaction between cue type and word type, $r(22)=-.51$, $p<.01$; and one involving the interaction between cue type, word type, and covering the keyboard, $r(22)=-.41$, $p<.05$. The correlation with cue type approached significance, $r(22)=-.39, p<.06$. The correlation with keyboard covered versus uncovered was not significant, $r(22)=-.29, p<.17$, Thus, covering the keyboard slowed typing rates by about the same amount, regardless of skill level, except for the highest-order interaction, whose magnitude decreased with skill. A similar analysis relating WPM to RT1 yielded no significant correlations.

Error rates were calculated as the percentages of words in which at least one error occurred. The error rate was higher when the hands were covered $(M=15.2 \%)$ than when they were not $(M=10.1 \%)$ and higher for unimanual words $(M=13.9 \%)$ than for bimanual words $(M=11.4 \%)$.

Table 1 Summary tables for ANOVAs on typing rates, response time to the first keystroke (RT1), response time to the space bar (SBRT), and error rate $(\mathrm{ER})$

\begin{tabular}{|c|c|c|c|c|c|c|c|c|c|c|c|c|}
\hline \multirow[t]{2}{*}{ Effect } & \multicolumn{3}{|c|}{ Typing Rate } & \multicolumn{3}{|l|}{ RT1 } & \multicolumn{3}{|l|}{ SBRT } & \multicolumn{3}{|l|}{ ER } \\
\hline & $M S E$ & $F$ & $\eta^{2} p$ & $M S E$ & $F$ & $\eta^{2} p$ & $M S E$ & $F$ & $\eta_{p}^{2}$ & $M S E$ & $F$ & $\eta^{2} p$ \\
\hline Keyboard (K) & 5,228 & $9.9^{*}$ & .301 & 31,255 & 0.6 & .027 & 20,306 & 2.5 & .099 & 133 & $9.8^{*}$ & .298 \\
\hline Cue $(\mathrm{C})$ & 9,916 & $81.0^{*}$ & .779 & 43,812 & $53.4^{*}$ & .699 & 4,429 & $21.1^{*}$ & .479 & 100 & 0.9 & .033 \\
\hline Word (W) & 6,430 & $29.3^{*}$ & .560 & 12,858 & $22.3^{*}$ & .493 & 7,994 & 0.6 & .026 & 37 & $7.9^{*}$ & .257 \\
\hline $\mathrm{K} \times \mathrm{C}$ & 3,230 & 1.1 & .045 & 8,276 & 0.7 & .031 & 8,356 & 0.8 & .034 & 57 & 0.4 & .017 \\
\hline $\mathrm{K} \times \mathrm{W}$ & 1,544 & 0.1 & .003 & 4,656 & $5.5^{*}$ & .193 & 9,189 & 0.1 & .005 & 23 & 0.1 & .003 \\
\hline $\mathrm{C} \times \mathrm{W}$ & 6,022 & $69.8^{*}$ & .752 & 19,897 & $19.8^{*}$ & .463 & 6,838 & $7.0^{*}$ & .233 & 32 & 0.1 & .002 \\
\hline $\mathrm{K} \times \mathrm{C} \times \mathrm{W}$ & 1,465 & 0.4 & .019 & 8,734 & $5.0^{*}$ & .178 & 7,862 & 0.7 & .031 & 17 & 0.0 & .002 \\
\hline
\end{tabular}

Degrees of freedom for each effect $=1,23 .{ }^{*} p<.05$. 
The difference between hand cues $(M=13.3 \%)$ and whole cues $(M=12 \%)$ was not significant, nor were any other effects. With hand cues, typists could make errors by typing a letter with the uncued hand or by typing a wrong letter with the cued hand. Covering the keyboard increased error rates in the uncued hand $(M \mathrm{~s}=4.3 \%$ vs. $2.1 \%), F(1,23)=$ $20.7, p<.01$, but not in the cued hand $(M \mathrm{~s}=10.1 \%$ vs. $8.3 \%), F(1,23)=2.7, p<.17$, suggesting that vision was important in avoiding uncued-hand errors.

\section{Discussion}

Our results demonstrate that attending to the hands disrupts skilled typing: typing rate, RT1, and SBRT were longer when typists typed only the letters assigned to one hand than when they typed normally, replicating Logan and Crump (2009). The novel contribution of our results is to demonstrate that vision is important in producing the disruption: The disruption was greater when we covered the hands and the keyboard than when we did not. This finding confirms the hypothesis that typists watch their hands to determine which hand types each keystroke and slow their rate of typing to allow enough time to inhibit keystrokes from the uncued hand. Logan and Crump (2009) proposed this hypothesis but did not test it directly. Our results suggest that typists may use kinesthesis as well as vision because they were able to respond to hand cues when we blocked vision. We did not test the role of kinesthesis directly, so this conclusion is more speculative.

Another novel contribution of our results is to demonstrate that vision is important in normal typing: covering the hands and keyboard slowed the typing of single words in the wholecue condition and the typing of paragraphs in the speed test. This suggests that modern typists depend on visual feedback to control their typing in the same way that professional typists of yesteryear did, because they responded in the same way to the same manipulation (Long, 1976; Rabbitt, 1978). Thus, the differences in training and experience do not seem to have produced differences in reliance on feedback.

Our results are consistent with Logan and Crump's $(2009,2010,2011)$ theory that typing is controlled by two nested feedback loops. The lower loop is modular (Fodor, 1983), translating words into sequences of keystrokes, assigning letters to hands and fingers, and executing keystrokes on the keyboard. It is grafted onto preexisting language and reading skills. It is informationally encapsulated (Fodor, 1983), so the higher loop does not know which hand is assigned to which letter. The higher loop interfaces with language comprehension and generation to translate ideas into sequences of words to be typed. It is central, in Fodor's terms, using many sources of information to do its work. Logan and Crump (2010) suggested that the higher loop recruits vision to detect errors in typewriting by monitoring the output that is echoed on the computer screen. Our results are consistent with this idea, suggesting that the higher loop also recruits vision to discover which hand types which letter. Logan and Crump (2009) hypothesized that vision would play this role. The novel contribution of the present research was to test and confirm that hypothesis.

Two alternative interpretations can be ruled out. First, typing might be controlled by a single loop that goes from language to keystrokes. The present results and the previous ones demonstrating paradoxical disruption of skilled typing by drawing attention to the hands are inconsistent with this interpretation (Logan \& Crump, 2009). Typists know but do not know which hand types which letter. The resolution to this paradox is to propose a hierarchical control system in which different parts of the hierarchy know different things (also see Crump \& Logan, 2010a, 2010b; Liu et al., 2010; Logan \& Crump, 2010; Shaffer, 1976).

Second, hand cues might slow typing because they require typists to break up (Jordan, 1995) and type unfamiliar sequences of keystrokes (Gentner, Larochelle, \& Grudin, 1988; Shaffer \& Hardwick, 1968). Typing unfamiliar sequences is unlikely to have caused the disruption, because Logan and Crump (2009) found no disruption when they colored right-hand letters red and left-hand letters green (or vice versa) and cued typists to type only red or green letters. The sequences were unfamiliar but were typed as quickly as intact words. Breaking up familiar sequences is unlikely to have caused the disruption, either. Hand cues disrupted typing of unimanual words, in which all letters were typed and no familiar sequences were disrupted (also see Logan \& Crump, 2009), so breaking up familiar sequences is not necessary to produce disruption. Moreover, skilled typists can easily stop typing in the middle of a word in response to a stop signal (Logan, 1982), so breaking up familiar sequences is not sufficient to produce disruption. It seems more likely that the disruption is produced by the requirement to make explicit some details of processing that are normally implicit (Logan \& Crump, 2009).

The hypothesis that typists slow their rates of typing to discover which hand types which letter can explain why typing is slower with hand cues than with whole cues. We believe it can also explain why typing is more error prone and why disruption is greater with bimanual than with unimanual words. The increased error rate follows from the assumption that the typing rate must slow down enough for typists to detect and inhibit keystrokes with the inappropriate hand. Inhibition takes time (Logan, 1982), so if typists do not slow down enough, they will fail to inhibit inappropriate keystrokes, which will increase the error rate.

The data suggest that typists do not know that all of the letters in unimanual words are typed with the same hand. 
Hand cues disrupted typing when all of the letters had to be typed, and SBRTs were longer when typists typed none of the letters $(M=2,044 \mathrm{~ms})$ than when they typed all of them $(M=$ $1,810 \mathrm{~ms}), t(23)=3.4, p<.01$. If they knew that all of the letters were typed in the same hand before they began typing, there should have been no disruption with unimanual words and SBRTs should have been very short when none of the letters had to be typed (also see Logan \& Crump, 2009).

This analysis explains why there was disruption for unimanual words, but it does not explain why there was more disruption for bimanual words. One possibility is that keystrokes are prepared in parallel although they are executed serially (Crump \& Logan, 2010a; Logan, Miller, \& Strayer, 2011; Rumelhart \& Norman, 1982). Often, the movement for the next keystroke begins before the movement for the current keystroke finishes (Flanders \& Soechting, 1992). Perhaps typists can see the beginning of the next keystroke as they are watching the current one. It may be easier to see successive keystrokes in the same hand than in different hands, particularly if the eyes are fixated on one hand. Further research will be necessary to evaluate this hypothesis.

\section{Conclusions}

We have shown that vision plays an important role in the disruption that is observed when skilled typists pay attention to the hands they use to type particular letters. The results are consistent with the hypothesis that typists slow their rates of typing so they can see which hand types which letter. We expect vision to play a similar role when attention to the details of performance disrupts other skills.

\section{References}

Beilock, S. L., Carr, T. H., MacMahon, C., \& Starkes, J. L. (2002). When paying attention becomes counterproductive: Impact of divided versus skill-focused attention on novice and experienced performance of sensorimotor skills. Journal of Experimental Psychology: Applied, 8, 6-16. doi:10.1037/1076-898X.8.1.6

Crump, M. J. C., \& Logan, G. D. (2010a). Hierarchical control and skilled typing: Evidence for word-level control over the execution of individual keystrokes. Journal of Experimental Psychology: Learning, Memory, and Cognition, 36, 1369-1380. doi:10.1037/a0020696

Crump, M. J. C., \& Logan, G. D. (2010b). Warning: This keyboard will deconstruct-The role of the keyboard in skilled typewriting. Psychonomic Bulletin \& Review, 17, 394-399. doi:10.3758/PBR.17.3.394

Davies, M. (2008). The corpus of contemporary American English (COCA): 410+ million words, 1990-present. Available online at www.americancorpus.org
Flanders, M., \& Soechting, J. F. (1992). Kinematics of typing: Parallel control of the two hands. Journal of Neurophysiology, 67, 12641274.

Fodor, J. A. (1983). The modularity of the mind: An essay on faculty psychology. Cambridge: MIT Press, Bradford Books.

Gentner, D. R., Larochelle, S., \& Grudin, J. (1988). Lexical, sublexical, and peripheral effects in skilled typewriting. Cognitive Psychology, 20, 524-548. doi:10.1016/0010-0285 (88)90015-1

James, W. (1890). The principles of psychology. New York: Henry Holt.

Jordan, M. I. (1995). The organization of action sequences: Evidence from a learning task. Journal of Motor Behavior, 27, 179-192.

Lashley, K. S. (1951). The problem of serial order in behavior. In L. A. Jeffress (Ed.), Cerebral mechanisms in behavior (pp. 112-136). New York: Wiley.

Liu, X., Crump, M. J. C., \& Logan, G. D. (2010). Do you know where your fingers have been? Explicit knowledge of the spatial layout of the keyboard in skilled typists. Memory \& Cognition, 38, 474484. doi:10.3758/MC.38.4.474

Logan, G. D. (1982). On the ability to inhibit complex movements: A stop-signal study of typewriting. Journal of Experimental Psychology: Human Perception and Performance, 8, 778-792. doi:10.1037/0096-1523.8.6.778

Logan, G. D., \& Crump, M. J. C. (2009). The left hand doesn't know what the right hand is doing: The disruptive effects of attention to the hands in skilled typewriting. Psychological Science, 20, 1296-1300. doi:10.1111/j.1467-9280.2009.02442.x

Logan, G. D., \& Crump, M. J. C. (2010). Cognitive illusions of authorship reveal hierarchical error detection in skilled typists. Science, 330, 683-686. doi:10.1126/science. 1190483

Logan, G. D., \& Crump, M. J. C. (2011). Hierarchical control of cognitive processes: The case for skilled typewriting. In B. H. Ross (Ed.), The psychology of learning and motivation: Advances in research and theory (Vol. 54, pp. 1-27). San Diego: Academic Press.

Logan, G. D., Miller, A. E., \& Strayer, D. L. (2011). Electrophysiological evidence for parallel response selection in skilled typists. Psychological Science, 22, 54-56. doi:10.1177/0956797610390382

Logan, G. D., \& Zbrodoff, N. J. (1998). Stroop-type interference: Congruity effects in color naming with typewritten responses. Journal of Experimental Psychology: Human Perception and Performance, 24, 978-992. doi:10.1037/0096-1523.24.3.978

Long, J. (1976). Visual feedback and skilled keying: Differential effects of masking the printed copy and the keyboard. Ergonomics, 19, 93-110.

Rabbitt, P. M. (1978). Detection of errors by skilled typists. Ergonomics, 21, 945-958. doi:10.1080/00140137808931800

Rumelhart, D. E., \& Norman, D. A. (1982). Simulating a skilled typist: A study of skilled cognitive-motor performance. Cognitive Science, 6, 1-36.

Salthouse, T. A. (1986). Perceptual, cognitive, and motoric aspects of transcription typing. Psychological Bulletin, 99, 303-319. doi:10.1037/0033-2909.99.3.303

Shaffer, L. H. (1976). Intention and performance. Psychological Review, 83, 375-393. doi:10.1037/0033-295X.83.5.375

Shaffer, L. H., \& Hardwick, J. (1968). Typing performance as a function of text. Quarterly Journal of Experimental Psychology, 20, 360-369. doi:10.1080/14640746808400175 